\title{
Estudio de la calidad del agua en la región centro de la meseta p’urhépecha
}

\section{Water quality study in the central region of the meseta p'urhépecha}

DOI: $10.46932 / \mathrm{sfjdv2n5-092}$

Received in: Oct 1st, 2021

Accepted in: Dec 30th, 2021

\section{Silvano Velázquez Roque \\ Maestro en Ciencias en Ingeniería Física, Cherán, Michoacán, México. \\ Tecnológico Nacional de México}

Instituto Tecnológico Superior P'urhépecha, División de Ingeniería Industrial. Carretera CarapanUruapan

Km. 31.5, Cherán Michoacán. C.P. 60270

E-mail: sil_roque@hotmail.com / silvano.rv@purhepecha.tecnm.mx

Miguel Ángel Madrigal Cardiel

Dr. En Ciencias de la Educación, Cherán, Michoacán, México.

Tecnológico Nacional de México

Instituto Tecnológico Superior P’urhépecha, División de Ingeniería Industrial. Carretera CarapanUruapan

Km. 31.5, Cherán Michoacán. C.P. 60270

E-mail: miguel.mc@purhepecha.tecnm.mx

Ulises Zurita Luna

Maestro en Ciencias en Ingeniería química, Cherán, Michoacán, México.

Tecnológico Nacional de México

Instituto Tecnológico Superior P’urhépecha, División en Energías Renovables. Carretera CarapanUruapan

Km. 31.5, Cherán Michoacán. C.P. 60270

E-mail: ulises.zl@purhepecha.tecnm.mx

Juan Damián Hernández

Ingeniero Industrial, Cherán, Michoacán, México.

Tecnológico Nacional de México

Instituto Tecnológico Superior P’urhépecha, División de Ingeniería Industrial. Carretera CarapanUruapan

Km. 31.5, Cherán Michoacán. C.P. 60270

E-mail: juan.dh@purhepecha.tecnm.mx

\begin{abstract}
In the present research work, a study was carried out on the quality of water for human consumption, in the central region of the P'urhépecha plateau of the state of Michoacán, Mexico, monitoring the variables of hardness, TDS, temperature, $\mathrm{pH}$ and concentration. of $\mathrm{As}$ and $\mathrm{Pb}$ in different sampling sites and in different seasons of the year, which were compared with parameters established by the Official Mexican Standard NOM-127-SSA1-1994, NOM-250-SSA1-2014, Environmental Protection Agency of the United States of America (USEPA) and those established by the World Health Organization (WHO). The results obtained show a pH that is $7 \pm 0.5$, the temperature oscillates between 18 and $22^{\circ} \mathrm{C}$, the hardness between
\end{abstract}


60 and $200 \mathrm{ppm}$, unlike a site that shows $600 \mathrm{ppm}$. SDT between 40 and $220 \mathrm{ppm}$ and a sample site of $400 \mathrm{ppm}$. On the other hand, the $\mathrm{Pb}$ concentration range was found between 0.15 and $0.5 \mathrm{ppm}$, while As concentrations ranged between 0.01 and $0.1 \mathrm{ppm}$, depending on the study site.

Keyword: water quality, hardness, TDS, lead, arsenic.

\section{RESUMEN}

En el presente trabajo de investigación se realizó un estudio sobre la calidad del agua para consumo humano, en la región centro de la meseta P'urhépecha del estado de Michoacán, México, monitoreando las variables de dureza, SDT, temperatura, $\mathrm{pH}$ y la concentración de As y $\mathrm{Pb}$ en diferentes sitios de muestreo y en diferentes estaciones del año, los cuales fueron comparados con parámetros establecidos por la Norma Oficial Mexicana NOM-127-SSA1-1994, NOM-250-SSA1-2014, Agencia de Protección Ambiental de los Estados Unidos de América (USEPA) y los establecidos por la Organización Mundial de la Salud (OMS). Los resultados obtenidos muestran un $\mathrm{pH}$ que se encuentra en $7 \pm 0.5$, la temperatura oscila entre los 18 y $22{ }^{\circ} \mathrm{C}$, la dureza entre los 60 y $200 \mathrm{ppm}$ a diferencia de un sitio que muestra $600 \mathrm{ppm}$. SDT entre 40 y $220 \mathrm{ppm}$ y un sitio de muestreo de $400 \mathrm{ppm}$. Por otra parte, el rango de concentración de $\mathrm{Pb}$ se encontró entre 0.15 y $0.5 \mathrm{ppm}$, mientras que las concentraciones de As oscilan entre 0.01 y $0.1 \mathrm{ppm}$, dependiendo del sitio de estudio.

Palabras claves: calidad del agua, dureza, SDT, plomo, arsénico.

\section{INTRODUCCIÓN}

Hablar de la calidad del agua para el consumo humano hoy en día es un tema de crucial importancia, debido que el vital líquido es un medio donde se llevan a acabo múltiples reacciones bioquímicas. Para el caso de las reacciones metabólicas en el ser humano, ésta permite el transporte de sustancias en forma iónica dentro de las células y entre ellas, lo que permite ser un medio indispensable para la vida humana (Voet, Voet \& Pratt, 2008). Para el caso de la agricultura su utilidad y aplicación resulta ser básica y en esta misma vertiente para la industria sus aplicaciones se diversifican en múltiples usos, dependiendo del giro de las mismas. Sin embargo, dada su utilidad de este compuesto vital se ha observado que cada vez se ve una mayor contaminación, debido a la combinación de diferentes especies químicas, por ejemplo: nitratos, cloruros, oloruros, elementos pesados entre varios otros, por ello es importante monitorear aspectos como la potabilidad, la presencia de materia orgánica dañina, la alcalinidad, cantidad excesiva de solidos disueltos, dureza, conductividad entre otras variables, (PerézLópez, E., 2016).

La calidad del agua por otro lado, implica parámetros permisibles que no dañen la salud en el cuerpo humano. Particularmente el agua para beber, es mayormente consumida si ésta pasa por un proceso de purificación. Una clasificación del agua para consumo humano es la siguiente: agua potable cruda y agua de mesa, donde la primera es agua clorada y con excedentes de sales, por otro lado, el agua de mesa, ha recibido un proceso de purificación a través de filtros de sedimentos, ósmosis inversa o cualquier otro 
proceso (Vidales-Olivos, 2000), el principal objetivos de dichos procesos es disminuir la cantidad de solidos disueltos, dureza y algunos otros parámetros establecidos por normas nacionales NOM-250SSA1-2014 e internacionales. Sin embargo, existen regiones de la república mexicana donde la población hace uso del vital líquido para beber directamente de manantiales y pozos profundos sin someterla a ningún proceso de purificación ni cloración.

Por otro lado, el agua que proviene de manantiales y pozos profundos puede contener algunos elementos pesados, como Arsénico, Plomo, Mercurio, Cadmio y varios otros elementos en forma de iones y sus diferentes compuestos, esto depende del subsuelo, minerales de contacto e incluso por contaminación de actividades por el ser humano, lo que implica un riesgo para la salud humana (efsa, 2015; Huang et. al, 2014).

En la región centro de la meseta P’urhépecha, ubicado en el estado de Michoacán, México-, se encuentran localizados los municipios de Nahuatzen, Cherán y Paracho, donde la población local extrae el agua de manera cotidiana de pozos profundos y manantiales consumiendo sin previa potabilización y/o purificación, por ello se vuelve importante la evaluación de la calidad del agua en fuentes de abastecimiento de los municipios de interés y de estudio de la región centro de la meseta P'urhépecha.

En esta investigación el objetivo principal es evaluar y determinar la calidad del agua en manantiales y pozos profundos ubicados en la región centro de la meseta P'urhépecha a través del análisis fisicoquímico y la determinación de metales pesados. Las variables para este estudio fueron: dureza, cantidad de solidos disueltos, $\mathrm{pH}$, temperatura, concentración de As (arsénico) y la concentración de $\mathrm{Pb}$ (plomo).

\section{MÉTODOS}

Para llevar a cabo esta investigación y el cumplimiento de los objetivos se establecieron los siguientes pasos:

a) Toma de muestras de agua de manantiales y pozo profundo.

Se identificaron doce sitios de muestreo; tres en el municipio de Nahuatzen, seis en el municipio de Cherán y tres en el municipio de Paracho, Se tomaron dos muestras por sitio en viales de $50 \mathrm{ml}$ en las diferentes estaciones del año.

b) Análisis fisicoquímico; dureza, cantidad de solidos disueltos, $\mathrm{pH}$ y temperatura.

Por cada muestra tomada en in situ se midió la dureza, solidos disueltos, $\mathrm{pH}$ y temperatura con equipos portátiles (electrodo de dureza, de TSD, pH-chimetro y termómetro laser).

c) Análisis fisicoquímico por emisión atómica por plasma para determinar metales pesados.

Las muestras tomadas fueron analizadas en un espectrómetro de emisión por plasmas modelo 4100 MP-AES marca Agillen.

d) Análisis estadístico de las zonas muestreadas. 
Una vez analizadas las muestras en sus diferentes variables por cada estación del año, se promedió el resultado y posteriormente se obtuvieron graficas en donde se aprecia de forma comparativa cada resultado de los manantiales y pozos profundos estudiados.

\section{RESULTADOS}

A continuación, se muestra una serie de gráficos en la que se puede apreciar el monitoreo y evolución de la cantidad del agua medida en ppm de las siguientes variables: dureza, cantidad de solidos disueltos, $\mathrm{pH}$, temperatura, concentración de $\mathrm{As}$ y la concentración de $\mathrm{Pb}$. Cabe resaltar que estos resultados dan información relevante de la calidad del agua de los diferentes sitios que fueron muestreados.

\section{$3.1 \mathrm{pH}$}

En la figura 1, se puede apreciar el $\mathrm{pH}$ promedio del agua de cada uno de los sitios donde fue muestreada en in situ. La mayoría de los pozos y manantiales se encuentra a un pH de 7 más, menos 0.5 unidades de diferencia entre algunos sitios. Este resultado se encuentra acorde con la norma permisible mexicana NOM-127-SSA1-1994 y la NOM-250-SSA1-2014, el cual establecen que el agua potable debe estar en un rango de pH entre 6.5-8.5. Cabe mencionar que la toma de muestras se realizó en diferentes temporadas del año y la variación que se encontró fue mínima (entre 0.4 y 0.5 ).

Figura 1. pH promedio del agua de zonas muestreadas.

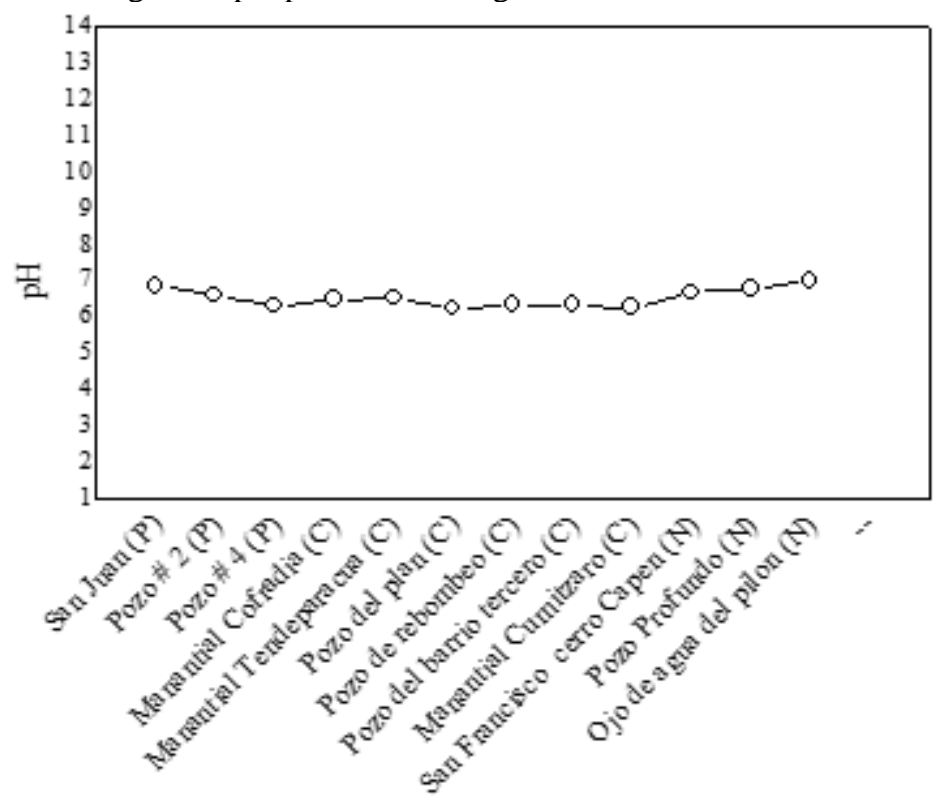




\subsection{TEMPERATURA}

Se encontró que la temperatura promedio varía según el sitio de muestreo. De acuerdo a la gráfica de la figura 2, los sitios de San Juan (P), Pozo \# 2 y Pozo \# 4 pertenecientes a la localidad de Paracho diferenciado por la letra $(\mathrm{P})$, el cual, mostraron un rango de temperatura entre $\operatorname{los} 19$ y $22{ }^{\circ} \mathrm{C}$, por otra parte, para los sitios de muestreo de la localidad de Cherán (diferenciado con la letra C): Manantial Cofradía (C), Manantial Tendeparacua (C), Pozo Plan (C), Pozo del Rebombeo (C), y Manantial Cumitzaro (C), la temperatura promedio es muy similar a la localidad de Paracho, es decir; que esta, entre los 18 y $21^{\circ} \mathrm{C}$, a diferencia del Pozo del Barrio Tercero (C) donde el promedio se encontró entre los 25 ${ }^{\circ} \mathrm{C}$, este mismo resultado fue similar al que se obtuvo con los sitios de la comunidad de Nahuatzen (diferenciados con la letra N), de los tres puntos de muestreo; San Francisco Cerro Capen (N), Pozo Profundo (N) y Ojo de agua del Plan.

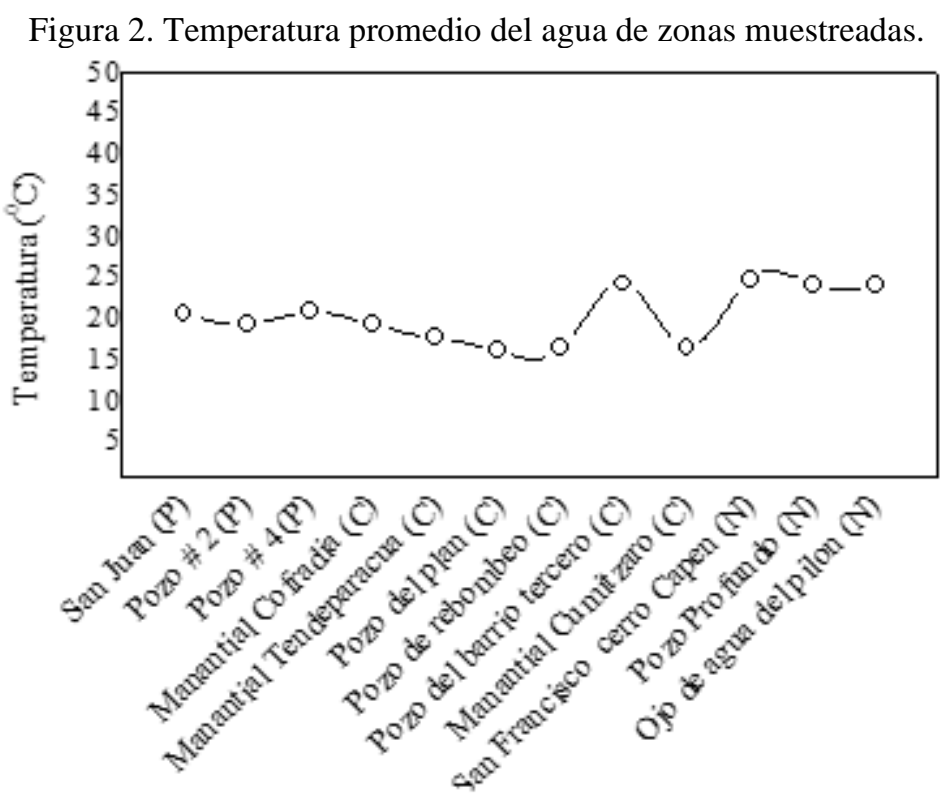

\subsection{DUREZA}

Cuando se habla de dureza se hace mención a la cantidad de carbonato de calcio $\left(\mathrm{CaCO}_{3}\right)$ disuelto en el agua en mayor concentración y en menor el carbonato de magnesio $\left(\mathrm{MgCO}_{3}\right)$. En este sentido el agua puede tener diferentes connotaciones, agua dura, agua parcialmente dura o blanda.

La Organización Mundial de la Salud (OMS), define la clasificación como agua blanda la que presenta concentraciones inferiores a $60 \mathrm{mg} / \mathrm{L}$ de Carbono de Calcio $\left(\mathrm{CaCO}_{3}\right)$, entre 61 y $120 \mathrm{mg} / \mathrm{L}$, moderadamente dura, entre 121 y $180 \mathrm{mg} / \mathrm{L}$ dura y aquella con valores superiores a $180 \mathrm{mg} / \mathrm{L}$ muy duras (Instituto Nacional de Epidemiológica y Microbiológica, 1992).

En este estudio en particular se tomó, en cuenta esta variable, puesto que es un parámetro fisicoquímico de importancia para la calidad del agua de consumo humano (potable). Para lo cual se 
encontraron los siguientes resultados. En los pozos de la localidad de Cheran la dureza, figura 3, muestra que se encuentran entre 80 y 100 ppm (o mg/L), para algunos sitios, mientras que para otro oscila entre los 100 y 150 ppm (manantial Tendeparacua), a diferencia del sitio conocido como Manantial Cumitzaro donde la concentración promedio de los carbonatos esta entre los 600 ppm.

Para las zonas de muestreo de Nahuatzen muestran un rango promedio entre los 60 y 100 ppm, La mayoría de los sitios de muestreo muestran que los resultados se encuentran dentro de la norma permisible establecidos por la NOM-250-SSA1-2014, el cual establece que la dureza debe estar a 500 ppm como límite máximo permisible. En contraste al sitio de muestreo del Manantial Cumitzaro del municipio de Cherán, donde arrojo valores por arriba de la norma, 600 ppm aproximadamente, esto indica un foco de atención para los habitantes que hacen uso de tal recurso hídrico. Por otro lado, en el municipio de Paracho en rango de los sitios de muestreados escila entre los 150 y 200 ppm.

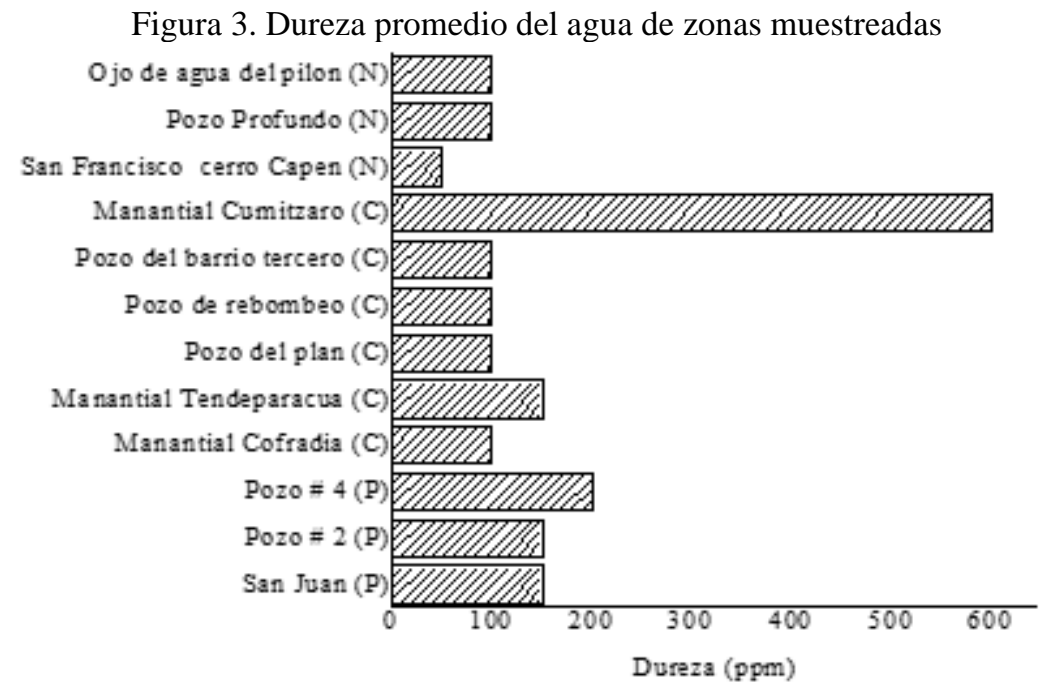

Por otro lado, la aceptación de la dureza varía dependiendo de la aceptación del público, incluso cuando se habla de agua embotellada para beber, esta depende de las especificaciones de calidad al cual están sometidas. Normalmente el rango de sabor del ión calcio esta entre los 100 a 300 ppm y el de magnesio es probablemente menor al de calcio. Según GEMS (1992) si la cantidad de magnesio es superior a 125 ppm en el agua de beber esta puede tener efectos laxantes. En este contexto si el exceso de carbonato de calcio en agua de beber está presente, esta puede generar cálculos renales en el organismo humano (Mora y Chamizo, 2007). Así mismo Melitón y Rosado (2008) concluyen que el consumo de agua con niveles por arriba de 120 ppm de carbonato de calcio son factores importantes en la producción de cálculos renales en las vías urinarias. Por otro lado, investigaciones realizadas mencionan que las aguas blandas y duras han sido relacionadas con problemas cardiacos (OMS, 1983) y cálculos renales (Evans y Taal, 2011; Mora y Alfaro, 1999). En acuerdo con los autores antes mencionados se muestran evidencia 
de la importancia que la dureza en agua potable es una variable que debe ser monitoreada de manera constante y más aún si el agua es de meza.

\subsection{SÓLIDOS DISUELTOS TOTALES (SDT)}

Los sólidos son partículas de compuestos que se encuentran diluidas en agua natural, se caracterizan por permanecer en estado sólido después de la evaporación del líquido. Básicamente existen de dos tipos sólidos suspendidos totales (SST) y los sólidos disueltos totales (SDT), correspondientes a residuos no filtrables y filtrables, respectivamente.

En la figura 4, se puede apreciar los resultados obtenidos de los sitios de muestreo, donde se muestra la cantidad promedio de SDT, para los sitios de la comunidad de Nahuatzen se observa que están en un rango de entre 60 y 90 ppm, mientras que para los de la comunidad de Cheran el rango se encuentra entre los 50 y 70 ppm a diferencia del sitio Manantial de Cumitzaro donde se muestra una cantidad promedio de 400 ppm. Por otro lado, en la localidad de Paracho, el rango de SDT está entre los 140 y 200 ppm. Cabe mencionar que estos resultados muestran evidencia que se encuentran dentro de la cantidad máxima permisible.

Figura 4. Promedio de sólidos disueltos en agua de las zonas muestreadas

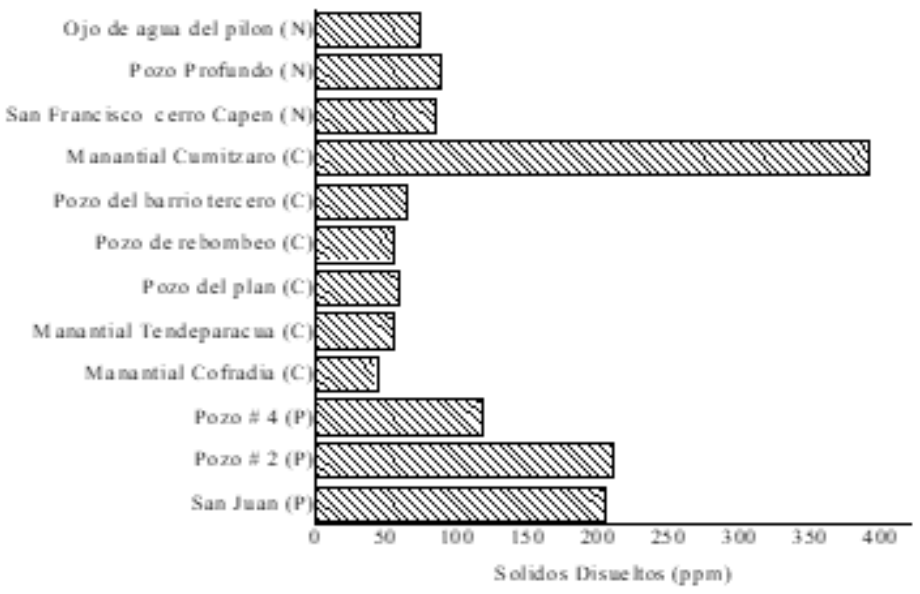

En contraste, la NOM-127-SSA1-1994 establece una máximo permisible de SDT de 1000 ppm (mg/L), mientras que la agencia de protección ambiental de los Estados Unidos de América (USEPA), sugiere un máximo de 500 ppm en agua potable, esto debido a que si la cantidad de SDT está arriba de lo sugerido, el agua puede presentar apariencia turbia y con sabor distinto, incluso las personas no acostumbradas al agua con alto contenido de SDT pueden experimentar irritación gastrointestinal al beber agua con esta característica (Sigler y Bauder, 2017). 


\section{5 $\mathrm{Pb}$ (Plomo)}

En la siguiente figura 5, se puede apreciar los resultados obtenidos de la concentración de $\mathrm{Pb}$ encontradas en los diferentes manantiales y pozos profundos de las localidades antes mencionadas.

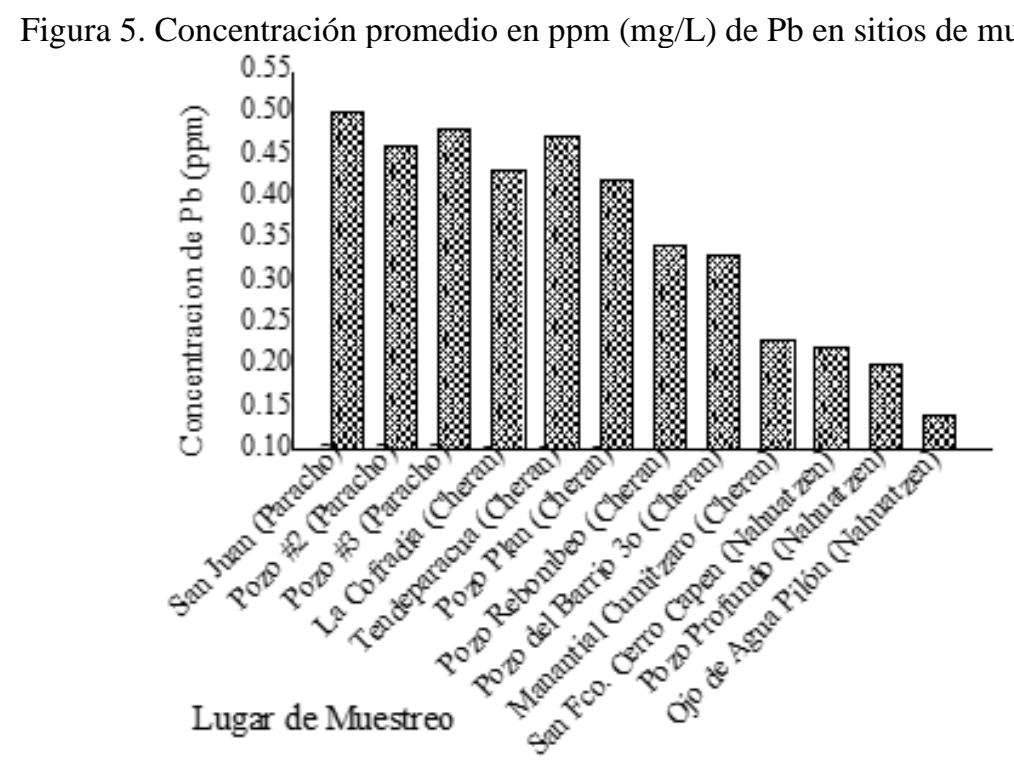

Los pozos profundos y manantiales de lo localidad de Paracho muestran concentraciones promedio de 0.46 y 0.5 ppm (mg/L), en los de Chéran se encuentran en un rango de 0.25 y 0.48 ppm, para los de la localidad de Nahuatzen entre 0.15 y 0.24 ppm.

La concentración de Pb ingerida en el organismo humano puede ocasionar severos daños a la salud, por esta razón la OMS y múltiples normas internacionales y naciones establecen una cantidad máximo permisible. La norma mexicana NOM-250-SSA1-2014, establece $0.01 \mathrm{mg} / \mathrm{L}(\mathrm{ppm})$ como máximo permisible contenida en agua de consumo humano. Si se observan los resultados de la gráfica anterior, estos muestran estar decenas de ppm por arriba de la norma establecida, el cual muestra que debe ser un tema de puntual atención por autoridades y organismos.

\subsection{As (Arsénico)}

El arsénico fue otro de los elementos al que se prestó puntual atención para su estudio y determinación en las manantiales y pozos profundos, en la figura 6, se puede observar la concentración encontrada en cada uno de los sitios muestreados. En los sitios de muestreo de la localidad de Paracho se encontró un rango promedio de 0.018 y 0.02 ppm. Para los de la comunidad e Cherán oscila entre 0.06 y $0.1 \mathrm{ppm}$ y en los de la localidad de Nahuatzen están en $0.1 \mathrm{ppm}$. Todos los sitios de muestreo revelan resultados por arriba de la norma permisible NOM-127-SSA1-1994, NOM-250-SSA1-2014 y la OMS el cual establecen una cantidad máxima de $0.01 \mathrm{mg} / \mathrm{L}$ ó $0.01 \mathrm{ppm}$. Si bien, no se han reportado resultados 
sobre el tema del arsénico y plomo en estos sitios y sus alrededores, cabe mencionar que la existencia de dichos metales y otros metaloides de interés pueden explicarse por varios factores presentes. La principal puede ser la presencia geológica, por ser una zona volcánica, por otro lado, investigaciones previas han reportado que el arsénico está presente en la atmosfera debido a fuentes naturales como reacciones ambientales, actividad biológica, emisiones volcánicas e incluso provenientes por un amplio rango de actividades antropogénicas (Campos et al., 2007; Alam et al., 2014) incluyendo la agricultura. Incluso el arsénico puede estar presente en el aire, el agua y el suelo a través de tormentas de polvo y agua de escorrentía, por lo que la contaminación de arsénico está muy extendida debido a su fácil dispersión (Gillispie et al., 2015).

Figura 6. Concentración promedio en $\mathrm{ppm}(\mathrm{mg} / \mathrm{L})$ de As en sitios de muestreo.

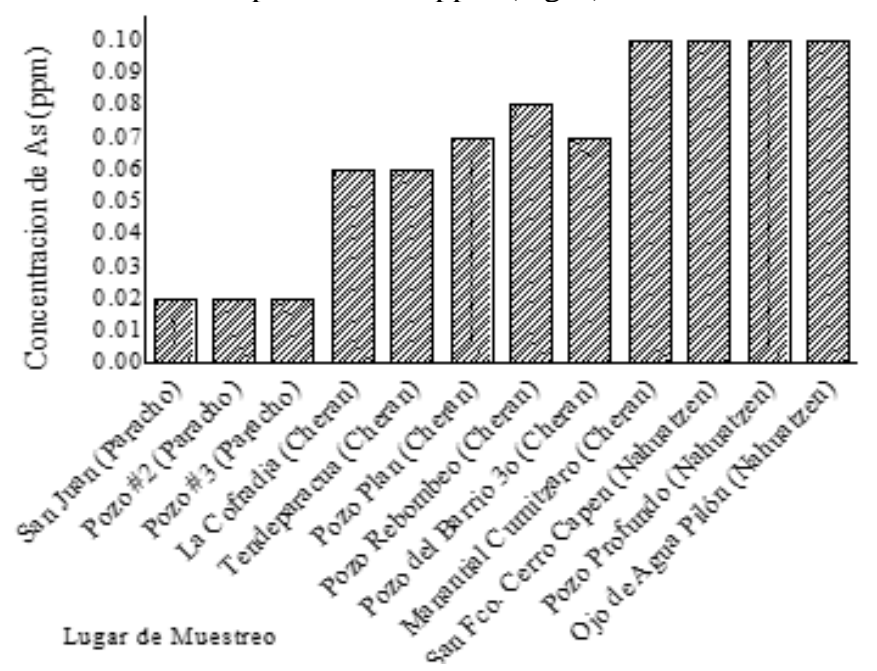

\section{CONCLUSIONES}

La calidad del agua para consumo humano es de vital importancia debido a su alta demanda y como medio de reacciones bioquímicas en el metabolismo celular. Por lo anterior se debe tener presente las normas vigentes nacionales e internacionales, para tomar en cuenta los parámetros permisibles en la calidad de agua de mesa que ayuden a mejorar la calidad de vida.

En base a los resultados obtenidos en esta investigación se puede concluir que:

-Se observó que las variables de dureza, SDT, $\mathrm{pH}$ y Temperatura se encuentran dentro de la norma establecida, NOM-127-SSA1-1994, NOM-250-SSA1-2014, la OMS e incluso por los establecido por la agencia de protección ambiental de los Estados Unidos de América. A diferencia de un sitio de muestreo en la comunidad de Cheran, el punto denominado manantial de Cumitzaro, la cantidad de dureza esta al rededor los 600 ppm y SDT está sobre los 400 ppm.

-Por el contrario, para el caso del Pb y As, éstas se encuentran por arriba de la norma NOM-127SSA1-1994, NOM-250-SSA1-2014. 


\section{BIBLIOGRAFÍA}

Alam, M. S., Y. Wu, and T. Cheng. 2014. Silicate minerals as a source of arsenic contamination in groundwater. Water, Air, Soil Pollut. 225: 2201-2215.

Campos, V., C. Valenzuela, M. Alcorta, G. Escalante, and M. Mondaca. 2007. Isolation of Arsenic resistance bacteria from volcanic rocks of Quebrada Camarones, Parina Region, Chile. Gayana 71: 150155.

European Food Safety Authority (efsa).2015. Recuperado de: http://www.efsa.europa.eu/en/topics/ topic/metals.

Evans, P. D., \& Taal, M. W. (2011). Epidemiology and causes of chronic kidney disease. Medicine, 39, 7. doi: 10.1016/j.mpmed.2011.04.007.

Instituto Nacional de Higiene Epidemiológica y Microbiología. (1992). Agua y Salud. La Habana, Cuba. Editorial Ciencias Médicas. Pág. 50.

Huang, Z. et al., (2014). Heavy metals in vegetables and the health risk to population in Zhejiang, China. Food Control, 36(1), pp.248-252. Available at: DOI: http://dx.doi.org/10.1016/j.foodcont.2013.08.036. GEMS.( 1992). Guía Operativa GEMS/Agua. Canadá. Tercera Edición. Capítulo 3,. Pág. 6-12

Gillispie, E. C., T. D. Sowers, O. W. Duckworth, and M. L. Polizzotto. 2015. Soil pollution due to irrigation with arsenic contaminated groundwater: Current state of science. Curr. Pollut. Rep. 1: 1-12.

Melitón, W. \& Rosado, F. (2008) "Impacto en la salud por consumo de agua dura en pobladores de la parte baja del río Chili, Arequipa, Perú". (Tesis de investigación). Universidad Nacional del Altiplano. Puno, Perú.

Mora Alvarado Darner y Chamizo García Horacio. (2007). Estudio exploratorio-ecológico sobre las concentraciones de sales de calcio en el agua para consumo humano y la litiasis renal en Costa Rica. Rev. costarric. salud pública vol.16 n.31 San José Dec. ISSN 1409-1429

Mora Alvarado Darner y Alfaro Herrera Nuria. (1999) Caracterización y distribución por cantones de la dureza del agua en las fuentes utilizadas para consumo humano en Costa Rica. Rev. costarric. salud pública vol.8, n.15, pp.01-15. ISSN 1409-1429.

Norma Oficial Mexicana NOM-250-SSA1-2014. (2014). Proyecto de norma oficial mexicana proy nom250-ssa1-2014, agua para uso y consumo humano. límites máximos permisibles de la calidad del agua y requisitos sanitarios que deben cumplir los sistemas de abastecimiento de agua públicos y privados, su control y vigilancia. procedimiento sanitario de muestreo. DOF. Recuperado de; http://www.dof.gob.mx/normasOficiales/5448/SALUD/SALUD.html

Norma Oficial Mexicana NOM-127-SSA1-1994. (1994). Salud ambiental, agua para uso y consumo humano-Límites permisibles de calidad y tratamientos a que debe someterse el agua para su potabilización. DOF. Recuperado https://www.gob.mx/cms/uploads/attachment/file/110534/NOM_127_SSA1_1994.pdf

OMS. (1983). "Calidad del Agua, Oligoelementos y Enfermedades Cardiovasculares. Ginebra, Crónicas de la OMS. No. 27. Pág. 572. 
Pérez-López, E. (2016). Control de calidad en aguas para consumo humano en la región occidental de Costa Rica. Tecnología en Marcha. Vol. 29, Nº 3. Pág 3-14. DOI: http://dx.doi.org/tm.v29i3.2884

Sigler, P. W. A., \& Bauder, J. (2017). Alcalinidad, pH, y Sólidos Disueltos Totales. 1.

Vidales Olivo, Amelia (2000). Diagnóstico de la Calidad de Agua de Mesa: Una Acción Positiva. Conciencia Tecnológica, (14),41-46. ISSN: 1405-5597. Recuperado de: https://www.redalyc.org/articulo.oa?id=94401409

Voet, D., Voet, J. \& Pratt, C. (2008). Fundamentos de bioquímica, la vida a nivel molecular. 2 ed. España: Editorial Panamericana. 